\title{
BMJ Open Choice of ANaesthesia for EndoVAScular treatment of acute ischaemic stroke at posterior circulation (CANVAS II): protocol for an exploratory randomised controlled study
}

\author{
Fa Liang, ${ }^{1}$ Yan Zhao, ${ }^{1}$ Xiang Yan, ${ }^{1}$ Youxuan $\mathrm{Wu},{ }^{1}$ Xiuheng $\mathrm{Li},{ }^{1}$ Yang Zhou, ${ }^{1}$ \\ Minyu Jian, ${ }^{1}$ Shu Li (D) , ${ }^{1}$ Zhongrong Miao, ${ }^{2}$ Ruquan Han, ${ }^{1}$ Yuming Peng (D) 1
}

To cite: Liang F, Zhao Y, Yan X, et al. Choice of ANaesthesia for EndoVAScular treatment of acute ischaemic stroke at posterior circulation (CANVAS II): protocol for an exploratory randomised controlled study. BMJ Open 2020;10:e036358. doi:10.1136/ bmjopen-2019-036358

- Prepublication history and additional material for this paper are available online. To view these files, please visit the journal online (http://dx.doi. org/10.1136/bmjopen-2019036358).

$\mathrm{FL}$ and $\mathrm{YZ}$ are joint first authors.

Received 12 December 2019

Revised 16 June 2020

Accepted 29 June 2020

D Check for updates

(C) Author(s) (or their employer(s)) 2020. Re-use permitted under CC BY-NC. No commercial re-use. See rights and permissions. Published by BMJ.

${ }^{1}$ Department of Anesthesiology, Beijing Tiantan Hospital, Capital Medical University, Beijing,

China

${ }^{2}$ Department of Interventional

Neurology, Beijing Tiantan Hospital, Capital Medical University, Beijing, China

Correspondence to

Yuming Peng;

florapym766@163.com

\section{ABSTRACT}

Introduction Observational and interventional studies indicate that the type of anaesthesia may be associated with the postprocedural neurological function in patients with anterior circulation acute ischaemic stroke undergoing endovascular treatment. Patients with acute posterior circulation ischaemic stroke may experience different physiological changes and result in severe neurological outcome. However, the effect of the type of anaesthesia on postprocedure neurological function remained unclear in this population.

Methods and analysis This is an exploratory randomised controlled trial that will be carried out at Beijing Tiantan Hospital, Capital Medical University. Patients with acute posterior circulation ischaemic stroke and deemed suitable for emergency endovascular recanalisation will be recruited in this trial. Eighty-four patients will be randomised to receive either general anaesthesia or conscious sedation with 1:1 allocation ratio. The primary endpoint is the 90-day modified Rankin Scale.

Ethics and dissemination The study has been reviewed by and approved by Ethics Committee of Beijing Tiantan Hospital of Capital Medical University (KY2017-074-02). If the results are positive, the study will indicate whether the type of anaesthesia affects neurological outcome after endovascular treatment of posterior stroke. The findings of the study will be published in peer-reviewed journals and presented at national or international conferences. Trial registration number NCT03317535.

\section{INTRODUCTION}

Acute ischaemic stroke (AIS) at posterior circulation account for $17 \%-60 \%$ of acute ischaemic stroke with difficulty in treatment, which result in a poor outcome and mortality rate of $80 \%-95 \% .{ }^{1-5}$ Early recanalisation is one of the most important predictors of favourable outcome for posterior circulation AIS patients at 90 days. ${ }^{6}$ Though endovascular therapy (EVT) has been demonstrated to be an effective and safe treatment with around $30 \%$ good clinical outcome improvement and

\section{Strengths and limitations of this study}

- This is the first randomised control study to observe the effect of anaesthesia modality on neurological outcome of patients with posterior circulation acute ischaemic stroke.

- This study involves strict randomised system, clear inclusion and exclusion criteria, a rigorous uniform protocol to balance intraoperative variables between groups.

- One limitation of the study is it is a single-centred trial, future multicentre trial is needed to verify the effects of anaesthesia on patients with posterior circulation acute ischaemic stroke undergoing endovascular treatment

- The sample size is relatively small and the result will need to be confirmed by larger-size trials.

- Patients are selected mainly based on time window, salvageable ischaemic brain tissue and initial infarct size might be considered in the inclusion criteria.

$35 \%$ mortality decrease at 90 days, there is a substantial proportion of patients with poor clinical outcomes even after timely successful reperfusion. ${ }^{7-10}$ Many factors may contribute to the mismatched association of high reperfusion rate and poor clinical outcome, including patient-specific factors (age, collaterals status, initial stroke severity, infarct volume and site, and distal artery emboli), procedural-specific factors (time from onset to recanalisation, degree of recanalisation) and anaesthesia management. ${ }^{211} 12$

The optimal anaesthesia choice and management for AIS patients during EVT, general anaesthesia (GA) or conscious sedation (CS), is still unclear. Benefits of GA with tracheal intubation or laryngeal mask include secured airway to avoid aspiration, body immobility to avoid vessel perforation and 
better digital subtraction angiography imaging. On the other hand, hypotension, nosocomial infection, delayed procedure initiation, loss of neurological evaluation and hyperventilation may contribute to poor outcomes. CS management permits neurological function assessment, shortens mean time from door to groin puncture and minimises haemodynamic changes associate with GA. However, the risk of aspiration and substantial movement during endovascular procedural are the main two disadvantages associated with $\mathrm{CS} .{ }^{13-17}$

Three randomised controlled trials have compared the neurological outcomes after EVT with GA or CS in anterior circulation. The Sedation versus Intubation for Endovascular Stroke TreAtment (SIESTA) trial reported similar National Institute of Health Stroke Scale (NIHSS) scores at 24 hours between GA and CS, but favourable outcomes, measured by modified Rankin Scale (mRS) was found in patients with GA 3 months after treatment as a secondary outcome measure. ${ }^{14}$ Similarly, the General Or Local anaesthesia in Intra Arterial THerapy (GOLIATH) trial reported similar brain infarct volume and favourable 90-day mRS score increased with GA. ${ }^{18}$ However, the Anaesthesia during Stroke (AnStroke) trial reported no difference between GA and CS in 90-day mRS. ${ }^{19}$ Recently, a meta-analysis using fixed-effects model by obtaining individual patient data from the above-three trials with blinded endpoint evaluation, indicated significantly different results in favour of the GA group $(\mathrm{COR}=1.58$, 95\% CI: 1.09 to 2.29). ${ }^{20}$ Nevertheless, these findings should be interpreted with caution. First, these trials only provided insight into choice of anaesthesia modality in the anterior circulation AIS population, the result of above researches is not appropriate for patients with posterior circulation occlusions. Second, only AnStroke trial analysed the neurological function at 90 days as the primary outcome, while in other two trials, it was analysed as a secondary outcome. Hence, the conclusion on relationship between anaesthesia and neurological function at 90 days should be drawn with caution. ${ }^{19}$

A few studies observed the feasibility of monitored anaesthesia care for elective endovascular procedures either in anterior or posterior circulations, and demonstrated high technical success with low rates of periprocedural complications and mortality. ${ }^{21}{ }^{22}$ Although these studies included a relatively large proportion of posterior circulation interventions and showed promising results about feasibility of monitored anaesthesia care, it is unreasonable to employ previous results in emergent setting, with potential presence of severe midbrain ischaemia. Moreover, only one study focused purely on the posterior circulation patients and investigated the influence of anaesthesia modality and management on clinical and angiographic outcomes. ${ }^{21}$ In this retrospective, matched, case-control study, CS was found to be feasible and appeared to be as safe and effective as GA. However, retrospective design and relatively limited sample size may introduce undetected biases. Furthermore, there is no published randomised controlled trial that explored whether GA or CS are associated with different neurological outcomes at 90 days in patients undergoing EVT for posterior circulation AIS.

On the basis of previous studies, we propose to conduct a trial to compare neurological outcome in posterior circulation AIS patients receiving GA with those receiving CS for EVT.

\section{METHODS}

The protocol has been prepared according to Standard Protocol Items: Recommendations for Interventional Trials. ${ }^{23}$ All trial procedures are summarised in table 1. For completed checklists, see online supplementary file 1 .

\section{Study design}

This is a randomised, parallel-group, exploratory trial with blinded endpoint evaluation to determine whether GA or CS produces different neurological outcomes in posterior circulation AIS patients undergoing EVT. Posterior circulation AIS patients will be enrolled from Beijing Tiantan Hospital, Capital Medical University from 2018 to 2020. This trial was approved by the Ethics Committee of Beijing Tiantan Hospital of Capital Medical University (KY2017-074-02). The participants flowchart is briefly illustrated in figure 1.

\section{Participants}

Posterior circulation AIS patients who deemed suitable for recanalisation of the culprit's vessels will be considered for recruitment in the study. The inclusion criteria are vertebral artery and/or basilar artery responsible for posterior circulation ischaemia confirmed by CT angiography (CTA)/magnetic resonance angiography (MRA), the modified Thrombolysis in Cerebral Infarction (mTICI) score $\leq 1$, age $\geq 18$ years, stroke onset to treatment time $\leq 24$ hours and modified Rankin score $\leq 2$ before onset.

Patients with unclear radiological image to identify infarction and vessel occlusion, with intracranial haemorrhage (ICH), anterior circulation occlusion, Glasgow coma score (GCS) $\leq 8$, NIHSS score $<6$ or $>30$, postcirculation Alberta Stroke Program Early CT Score (pc-ASPECTS) $<6$, pons-midbrain index $\geq 3$, severe agitation or seizures, loss of airway protective reflexes and/ or vomiting on admission, intubated before EVT, unconsciousness and known allergy to anaesthetics or analgesics will be excluded from the study. Patients whose legal relative refuses to participate will be excluded. Both the neuroradiologists and the attending anaesthesiologist must agree that the patient is suitable with either GA or CS management before recruiting. Reasons why eligible patients are not recruited to the trial will be documented.

\section{Randomisation and blinding}

Randomisation occurs on the time of EVT when patients are admitted to the interventional neuroradiology suite, the decision for EVT has been made and written 


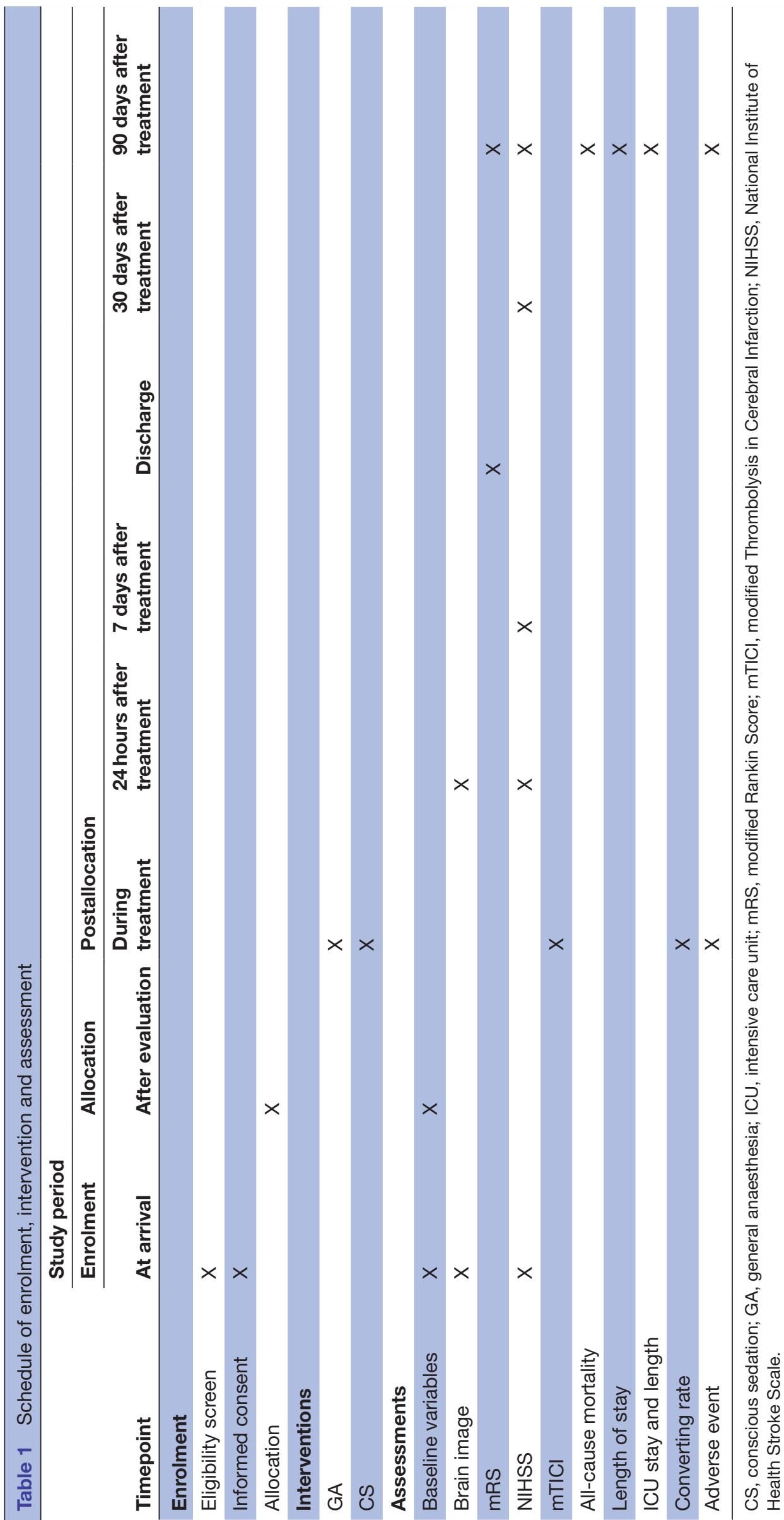

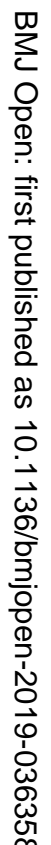

은

$\stackrel{\omega}{\underline{\omega}}$

¿

N

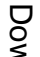

응

음

옥

룽

흠.

익

훙

옹

옹

을

๙ั

N

Q

员

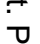

웅

융 


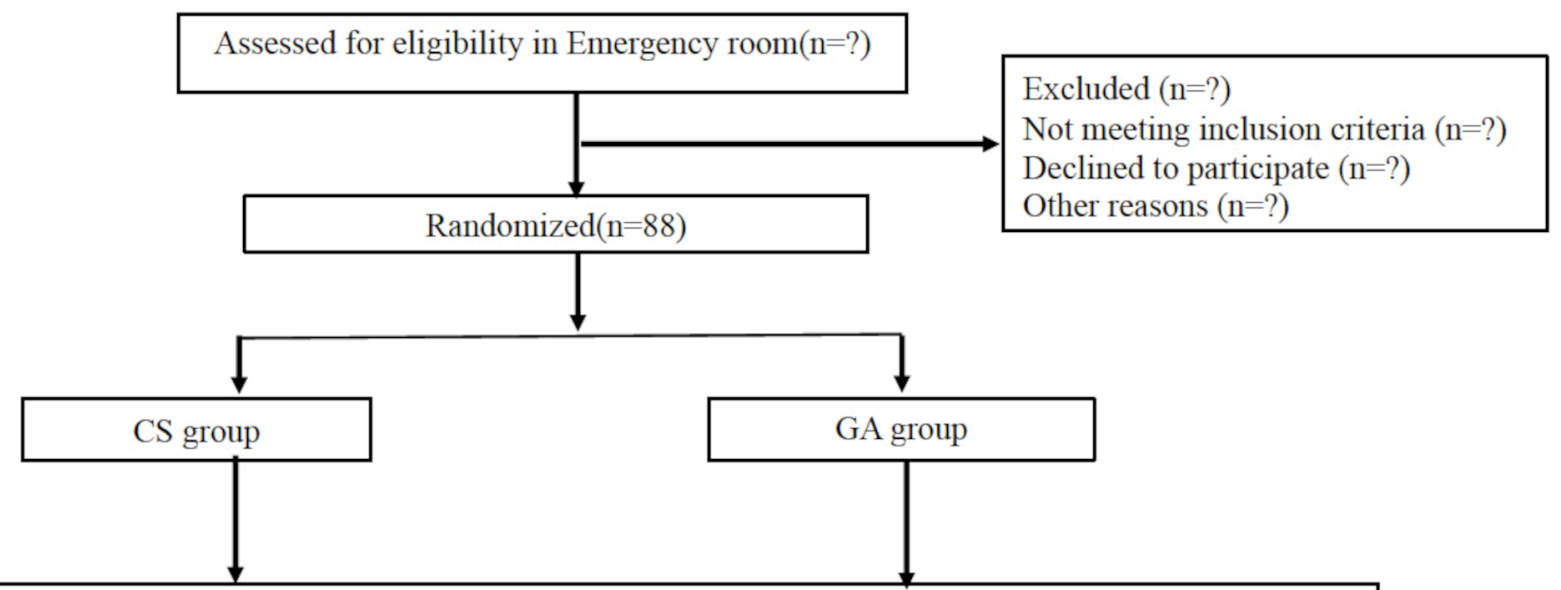

Assessment time:24h,7 days,30 days and 90 days after endovascular treatment

Outcome:

- Primary outcome: mRS score at 90-day after EVT,

- Secondary outcome: Change in NIHSS , mTICI, All-cause mortality, incidence of complications, length of stay, conversion rate, adverse events

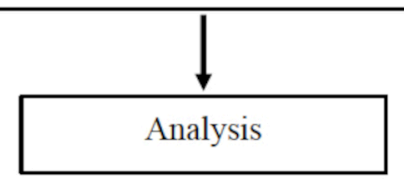

Figure 1 Consolidated Standards of Reporting Trials flow diagram for CANVAS II clinical trial. CS, conscious sedation; EVT, endovascular therapy; GA, general anaesthesia; mRS, modified Rankin Scale; mTICI, modified Thrombolysis in Cerebral Infarction; NIHSS, National Institute of Health Stroke Scale.

informed consent (online supplementary file 2) is obtained from patient's legal representatives. Randomisation will be conducted via a computer-generated table. Patients will be randomly allocated to receive either GA or CS in a 1 to 1 ratio. A designated staff who will neither be involved in anaesthesia management nor follow-up will perform recruitment as well as allocation randomisation sequence. This designated staff will implement the allocation sequence through opaque, sealed and stapled envelopes. The endpoint assessors will be blinded to the randomisation group.

Standard operating procedures are applied to both groups to ensure no principal differences generated and uniform protocol implemented. Patients in both groups will receive local anaesthesia (LA) at puncture site, with $3-5 \mathrm{~mL}$ of $1 \%$ lidocaine hydrochloride prior to arterial puncture. The outcome assessors are blinded to the information of treatment for the enrolled patients and will evaluate the outcome variables for this study to ensure unbiased reporting. The anaesthesiologist, neuroradiologist as well as attending doctors in neurological intensive care unit will not be blinded as they need to participate in the safe administration of GA or CS and related medical care. The enrolled patients and his/her legal representatives will not be blinded, either.

\section{Standard anaesthesia management protocols during EVT (concomitant treatment)}

All randomised patients will receive standard monitoring, including ECG, non-invasive blood pressure (BP), heart rate, pulse oxygen saturation $\left(\mathrm{SpO}_{2}\right)$, invasive arterial pressure monitoring on radiologist arterial access line, arterial partial pressure of carbon dioxide $\left(\mathrm{PaCO}_{2}\right)$, endtidal carbon dioxide $\left(\mathrm{ETCO}_{2}\right)$, inspired oxygen fraction $\left(\mathrm{FiO}_{2}\right)$ and blood glucose. All patients will receive bispectral index (BIS) monitoring to assess the depth of sedation or anaesthesia with BIS probe placed on the forehead. Physiologic parameters will be recorded using purposely designed data collection table. BP and blood glucose will be controlled according to current guidelines for stroke therapy. ${ }^{24-26}$ Specifically, systolic BP is aimed to be kept between 140 and $180 \mathrm{~mm} \mathrm{Hg}$ and diastolic BP less than $105 \mathrm{~mm} \mathrm{Hg}$, with vasopressor support if necessary. Plasma glucose will be maintained at level of $140-180 \mathrm{mg} / \mathrm{dL}$ while $\mathrm{SpO}_{2}$ is aimed to be over $94 \%$, with $\mathrm{FiO}_{2}$ at a range from $40 \%$ to $60 \% .{ }^{27}$ It is anticipated that patients in the CS group may deteriorate during EVT and may, therefore, require endotracheal intubation or laryngeal mask insertion for airway protection. ${ }^{17}$ All anaesthesia-related treatment will be performed by anaesthesiologists of ischaemia stroke team.

\section{Interventions}

GA and CS in this trial are defined according to the practice guidelines for sedation and analgesia by nonanaesthesiologists due to the continuum ranging of level of sedation from minimal sedation to GA. ${ }^{28}$ Both GA and $\mathrm{CS}$ will be monitored and applied by anaesthesiologist. In CS group, patients will receive sedative drugs and follow the sedation protocol as following: bolus propofol of 
$0.3-0.5 \mathrm{mg} / \mathrm{kg}$, following continuous propofol infusion of $1-2 \mathrm{mg} / \mathrm{kg} / \mathrm{h}$ and remifentanil infusion of $0.01-0.06 \mathrm{ug} /$ $\mathrm{kg} / \mathrm{min} . \mathrm{SpO}_{2}$ will be kept above $94 \%$ with $40 \%-60 \%$ inhaled oxygen at $3 \mathrm{~L} / \mathrm{min}$ flow and $\mathrm{ETCO}_{2}$ is monitored via anaesthetic gas sample line at nasal vestibule and kept normocapnia. The BIS value will be maintained above 70 via adjusting the infusion of sedative drugs.

In GA group, patients will receive rapid sequence induction with endotracheal intubation or laryngeal mask insertion with propofol, remifentanil and muscle relaxant. Anaesthesia will be induced with infusion of propofol of $1-2 \mathrm{mg} / \mathrm{kg}$ and remifentanil of $0.2-0.8 \mathrm{ug} /$ $\mathrm{kg}$ for anaesthesia induction. ${ }^{27}$ Muscle relaxation will be achieved with rocuronium $(0.6 \mathrm{mg} / \mathrm{kg})$. After endotracheal intubation or laryngeal mask insertion, suction will be performed to mitigate risk of aspiration. Mechanical ventilation will be initiated to achieve normocapnia $\left(\mathrm{ETCO}_{2}\right.$ between 35 and $45 \mathrm{~mm} \mathrm{Hg}$ ) with a $40 \%-60 \%$ fraction of inspired oxygen. Anaesthesia will then be maintained with propofol $(4-6 \mathrm{mg} / \mathrm{kg} / \mathrm{h})$ and remifentanil $(0.05$ to $0.1 \mathrm{ug} / \mathrm{kg} / \mathrm{min})$ infusion to maintain the BIS value between 40 and 60 .

Converting from CS to GA is an important issue in this trial. In cases of procedural emergency including vessel perforation, ICH, subarachnoid haemorrhage, seizures, deep coma, GCS decrease to or less than 8 , respiratory failure $\left(\mathrm{ETCO}_{2} \geq 60 \mathrm{~mm} \mathrm{Hg}\right.$, or $\mathrm{SpO}_{2}<94 \%$ without relevant improvement by increasing inhaled oxygen fraction) and severe disturbance of the treatment procedure (vomiting, substantial movement and uncoordinated dysphoria), CS will be converted to GA. The decision to convert from CS to GA will be made by the neuroradiologist in charge and the attending anaesthesiologist. The number of patients and reasons for the conversion will be recorded in detail. Criteria for converting from CS to GA is listed in box 1 .

\section{Measurements}

All patients will be regularly visited while in hospital by outcome assessors who are blinded to the treatment allocation. The incidence of complications, including myocardial infarction, non-fatal cardiac arrest, new stroke, pulmonary embolism and deep venous thrombosis

Box 1 Griteria for converting from conscious sedation to general anaesthesia

1. Unconscious.

2. Glascow Coma Scale decrease to or less than 8.

3. Increase of end-tidal carbon dioxide $\left(\mathrm{ETCO}_{2}\right) \geq 60 \mathrm{~mm} \mathrm{Hg}$ and/or a decrease in pulse oxygen saturation $\left(\mathrm{SpO}_{2}\right)<94 \%$ despite oxygen supplementation.

4. Agitation that cannot be controlled with sedation and/or restraint.

5. Seizure attack.

6. Vomiting.

7. Recognised complications from endovascular therapy, such as vessel perforation leading to intracerebral haemorrhage or subarachnoid haemorrhage. will be recorded. All patients will receive brain imaging, including CTA, MRA before, 24 hours, 7 days (or at discharge, whichever sooner), 30 days and 90 days after randomisation. Brain image will be used to assess new brain haemorrhage (if present) and infarct volume. Efficacy of vessels recanalisation will be assessed by mTICI scale. The severity of stroke will be assessed using the NIHSS scale during the same period. Disability will be rated at discharge and 3 months after EVT and rated using mRS. A glossary of measurements is shown in online supplementary file 3 . To minimise loss of follow-up after discharge, study coordinators will contact the patients and his/her next-of-kin on a weekly basis. Besides, adverse effect will be also recorded.

\section{Study objectives}

The study aims to detect the difference of the postprocedural neurological function in patients with posterior circulation AIS under GA and CS, and hence to observe the effect of anaesthesia type on outcomes after EVT.

\section{Primary endpoint}

The primary endpoint is the neurological disability at 90 days after EVT measured by mRS, which ranges from 0 (no symptoms) to 5 (severe disability), and a favourable neurological outcome is defined as no symptom or no significant disability with $\mathrm{mRS} \leq 2 .{ }^{3} 152930$ The score will be evaluated by outcomes assessor who are blinded to allocation.

\section{Secondary endpoints}

The secondary endpoints include the followings:

1. Change in NIHSS, from baseline to 24 hours, 7 days (or at discharge), 30 days and 3 months after randomisation.

2. The score of mTICI will be evaluated before and after endovascular treatment.

3. All-cause mortality up to 3 months after randomisation.

4. The incidence of complications up to 3 months after randomisation.

5. The length of stay in the hospital and in intensive care unit after randomisation.

6. The rate of conversion from CS to GA.

7. Work-flow time, including door to door, door to groin puncture, puncture complete, groin puncture to recanalisation and treatment time.

8. All adverse events associated with this study will be recorded.

\section{Data Monitoring Committee}

The project will be monitored by a Data Monitoring Committee (DMC) composed of specialists in anaesthesiology, ethics, statistics and methodology. The DMC will audit through regular interviews or telephone calls. The DMC are responsible for terminating the research in case of severe adverse events. 


\section{Statistical analysis plan}

Descriptive statistics will be reported as means with SD and medians with IQR for normally distributed data and skewed continuous data, respectively, and counts (percentage) for categorical data. The data will be analysed on intention to treat and per protocol, however, the conclusion will be drawn according to the intention-to-treat analysis. The intention-to-treat analysis will depend on the allocated population while the per-protocol analysis will depend on the actual anaesthesia method the population receive. Differences in the primary endpoint will be compared between groups using Cochran-Mantel-Haenszel test. Furthermore, primary outcome will be analysed in following subgroups: age, gender, baseline NIHSS score; time from onset of stroke to EVT, site of arterial occlusion and mTICI score. Other categorical variables will be analysed by $\chi^{2}$ test and continuous variables using Student-t test or Mann-Whitney U test.

To allow for a varying number of follow-up measurements, the repeated measure analysis of variance methods with a mixed-model approach (treating time as a random effect and other covariates as fixed effects) will be utilised, and the specific comparison of change in each of those measurements between baseline and any specific postbaseline time point can be tested using linear contrast. In addition, missing data will be imputed using inverse probability weighting and the worst-case imputation scenarios. STATA V.14.0 for windows will be used for all statistical analyses. The statistical significance will be declared at type I error of 0.05 .

\section{Sample size calculation}

The PASS V.15 software (NCSS, LLC, USA) is used to calculate the sample size based on the primary endpoint-favourable outcome (mRS 0-2) at 3 months after randomisation. Several trials focused on the neurological outcome of patients with posterior circulation AIS, however, only two indicated the association between anaesthesia type and neurological outcome. In the case-control study of Jadhav et al, they reported the incidence of mRS $\leq 2$ at 90 days was $38.3 \%$ in CS and $31.1 \%$ in GA. ${ }^{21}$ On the other hand, a retrospective observational study in our institution reported a higher incidence of favourable neurological outcome at 90 days in CS compared with GA group (68.7\% vs $35.6 \%) .{ }^{31}$ However, other factors including preoperative NIHSS score, preoperative intravenous thrombolysis treatment confound the results validity. ${ }^{31}$ Meanwhile, in previous anterior circulation research, AIS patients receiving GA presented a favourable or similar neurological outcome at 90 days compared with CS. ${ }^{13} 1932$ Taking this into account, we consider that the sample size to detect $30 \%$ difference in mRS 0-2 would require 44 in each group to achieve power of $80 \%$ at a two-tailed significant level of 0.05 , with a drop-out rate of $5 \% .^{33}$

\section{Reporting of adverse events}

All adverse events associated with this trial will be recorded and closely monitored until resolution or stabilisation or until it has been shown that study treatment is not the cause of the event. The principal investigator is responsible for reporting all adverse events. Once adverse events occur, it should be immediately reported to the research department and informed to the principal investigator to determine the severity of the adverse events. All adverse events associated with this study will be recorded and reported to the Ethics Committee within 24 hours.

\section{Protocol amendment}

The chief investigator will be responsible for any decision to amend the protocol. If there is any modification (eg, changes to eligibility criteria, outcomes, analyses), the principle investigator will communicate and gain approval from the Ethical Committee of Beijing Tiantan Hospital, Capital Medical University prior to implementation and communicate with relevant parties.

\section{Ethics and dissemination}

The approval for the study was certificated by the Ethical Committee of Beijing Tiantan Hospital, Capital Medical University on 19 December, 2017 (reference number: KY2017-074-02). The study recruited the first patient on 1 February, 2018, and the estimated study completion date will be 31 December 2020. The findings of the study will be published in peer-reviewed journals and will be presented at national or international conferences.

\section{DISCUSSION}

This is an exploratory controlled randomised study aiming to detect the effect of anaesthesia choice on the neurological outcome in the patients with posterior circulation AIS undergoing EVT. The study aims to test the hypothesis that GA and CS have different effects on the postendovascular procedure neurological outcomes in patients with posterior circulation AIS.

Anaesthetic selection and peri-procedural management could be associated with outcomes in patients with posterior circulation AIS undergoing EVT. The haemodynamics disturbance and the changes in carbon dioxide tension may be associated with the outcomes. However, no consistent agreement about the anaesthesia modality has been reached, the anaesthetic protocols vary among different stroke centres. In daily clinical work, the actual practice of anaesthesia for EVT, especially for posterior circulation AIS patients, largely rely on local protocols and individual preference of neuroradiologists or anaesthesiologists. CS may be used for cooperative patients and GA for severely agitated, non-conscious and unprotected airway patients. ${ }^{13}$ A small number of retrospective studies (including patients with posterior circulation AIS) show that CS groups have better clinical outcomes, lower complication rate, shorter reperfusion time and less changes in haemodynamics and 
respiration. ${ }^{21} 2231$ In contrary, GA groups could suffer from worse neurological function, lower BP, aspiration pneumonia and/or high mortality. ${ }^{34-41}$ A recent retrospective, matched, case-control study of patients with posterior circulation AIS is the only study to explore the effect of anaesthesia management on outcome of patients with posterior AIS, however, great limitation and drawbacks in terms of study design, including selection bias and information bias impede the credibility of the results. ${ }^{21}$ Several confounding factors contribute to inconclusive results, including specific information of peri-interventional management, such as $\mathrm{BP}$, partial pressure of carbon dioxide and strict uniform anaesthesia protocol. Therefore, a prospective, randomised, controlled trial is required to account for the periprocedural confounders and demonstrate the effects of anaesthesia (type and management) on outcomes for patients with posterior AIS undergoing EVT.

This trial aims to explore the effect of anaesthesia choice on the postendovascular procedure outcomes in patients with posterior circulation AIS using a randomised controlled trial design. The features of the current study involve strict randomised system, clear inclusion and exclusion criteria, a rigorous uniform protocol to manage haemodynamic, respiratory parameters and blood glucose in GA and CS groups, and fulltime attending anaesthesiologists in each procedure. The findings of the study would contribute to serve as a reference for a future multicentre trial to verify the effects of anaesthesia on patients with posterior circulation AIS undergoing EVT.

\section{Patient and public involvement}

Patients and the public were not directly consulted in the development of the research question or outcome measures. Patients were not involved in the design, the recruitment and conduct of the study. At the completion of this trial, a manuscript will be prepared to present the trial results. Results of the final study will be disseminated to all study participants through their preferred method of communication indicated at the time of enrolment.

Contributors FL and YZ conceived the study. FL, YZ, SL, ZM, YP and RH initiated the study design and helped with protocol development and implementation. $\mathrm{FL}, \mathrm{XY}$, $X L Y W, Y Z$ and $M J$ helped in data collection and manuscript revision. YP and RH are the grant holders. FL and $\mathrm{YZ}$ are the co-first authors. $\mathrm{YP}$ is the responsible author. All authors contributed to refinement of the study protocol. All authors have read and approved the final manuscript.

Funding The trial is supported by the Beijing Municipal Administration of Hospitals of Ascent Plan (Grant No. DFL20180502), Beijing Municipal Administration of Hospitals Clinical Medical Development of Special Funding Support (Grant No. ZYLX201708) and Beijing Municipal Science and Technology Commission (Grant No. Z191100006619068)

Competing interests None declared.

Patient and public involvement Patients and/or the public were not involved in the design, or conduct, or reporting, or dissemination plans of this research.

Patient consent for publication Not required.

Provenance and peer review Not commissioned; externally peer reviewed.
Open access This is an open access article distributed in accordance with the Creative Commons Attribution Non Commercial (CC BY-NC 4.0) license, which permits others to distribute, remix, adapt, build upon this work non-commercially, and license their derivative works on different terms, provided the original work is properly cited, appropriate credit is given, any changes made indicated, and the use is non-commercial. See: http://creativecommons.org/licenses/by-nc/4.0/.

\section{ORCID iDs}

Shu Li http://orcid.org/0000-0002-5625-067X

Yuming Peng http://orcid.org/0000-0002-2630-2467

\section{REFERENCES}

1 Raymond S, Rost NS, Schaefer PW, et al. Patient selection for mechanical thrombectomy in posterior circulation emergent largevessel occlusion. Interv Neuroradiol 2018;24:309-16.

2 Luo G, Mo D, Tong X, et al. Factors associated with 90-day outcomes of patients with acute posterior circulation stroke treated by mechanical thrombectomy. World Neurosurg 2018;109:e318-28.

3 Lee YY, Yoon W, Kim SK, et al. Acute basilar artery occlusion: differences in characteristics and outcomes after endovascular therapy between patients with and without underlying severe atherosclerotic stenosis. AJNR Am J Neuroradiol 2017;38:1600-4.

$4 \mathrm{Kim}$ YW, Hong JM, Park DG, et al. Effect of intracranial atherosclerotic disease on endovascular treatment for patients with acute vertebrobasilar occlusion. AJNR Am J Neuroradiol 2016:37:2072-8.

5 Gao F, Lo WT, Sun X, et al. Combined use of mechanical thrombectomy with angioplasty and stenting for acute basilar occlusions with underlying severe intracranial vertebrobasilar stenosis: preliminary experience from a single Chinese center. AJNR Am J Neuroradiol 2015;36:1947-52.

6 Mokin M, Sonig A, Sivakanthan S, et al. Clinical and procedural predictors of outcomes from the endovascular treatment of posterior circulation strokes. Stroke 2016;47:782-8.

7 Gory B, Eldesouky I, Sivan-Hoffmann R, et al. Outcomes of stent retriever thrombectomy in basilar artery occlusion: an observational study and systematic review. J Neurol Neurosurg Psychiatry 2016;87:520-5.

8 van Houwelingen RC, Luijckx G-J, Mazuri A, et al. Safety and outcome of intra-arterial treatment for basilar artery occlusion. JAMA Neurol 2016;73:1225-30.

9 Huo X, Gao F, Sun X, et al. Endovascular mechanical thrombectomy with the solitaire device for the treatment of acute basilar artery occlusion. World Neurosurg 2016;89:301-8.

10 Singer OC, Berkefeld J, Nolte $\mathrm{CH}$, et al. Mechanical recanalization in basilar artery occlusion: the ENDOSTROKE study. Ann Neurol 2015;77:415-24.

11 Brinjikji W, Murad MH, Rabinstein AA, et al. Conscious sedation versus general anesthesia during endovascular acute ischemic stroke treatment: a systematic review and meta-analysis. AJNR Am J Neuroradiol 2015;36:525-9.

12 Yeo LLL, Holmberg A, Mpotsaris A, et al. Posterior Circulation Occlusions May Be Associated with Distal Emboli During Thrombectomy : Factors for Distal Embolization and a Review of the Literature. Clin Neuroradiol 2019;29:425-33.

13 Simonsen CZ, Sørensen LH, Juul N, et al. Anesthetic strategy during endovascular therapy: General anesthesia or conscious sedation? (GOLIATH - General or Local Anesthesia in Intra Arterial Therapy) A single-center randomized trial. Int J Stroke 2016;11:1045-52.

14 Schönenberger S, Möhlenbruch M, Pfaff J, et al. Sedation vs. Intubation for Endovascular Stroke TreAtment (SIESTA) - a randomized monocentric trial. Int J Stroke 2015;10:969-78.

15 Brinjikji W, Pasternak J, Murad MH, et al. Anesthesia-Related outcomes for endovascular stroke revascularization: a systematic review and meta-analysis. Stroke 2017;48:2784-91.

16 Kılıç Y, Baș Sema Șanal, Aykaç Özlem, et al. Nonoperating room anesthesia for interventional Neuroangiographic procedures: outcomes of 105 patients. J Stroke Cerebrovasc Dis 2020:29:104495.

17 Ozhan MO, Eskin MB, Atik B, et al. Laryngeal mask airway for general anesthesia in interventional neuroradiology procedures. Saudi Med J 2019:40:463-8.

18 Simonsen CZ, Yoo AJ, Sørensen LH, et al. Effect of general anesthesia and conscious sedation during endovascular therapy on infarct growth and clinical outcomes in acute ischemic stroke: a randomized clinical trial. JAMA Neurol 2018;75:470-7.

19 Löwhagen Hendén P, Rentzos A, Karlsson J-E, et al. General anesthesia versus conscious sedation for endovascular treatment of 
acute ischemic stroke: the AnStroke trial (anesthesia during stroke). Stroke 2017;48:1601-7.

20 Schönenberger S, Hendén PL, Simonsen CZ, et al. Association of general anesthesia vs procedural sedation with functional outcome among patients with acute ischemic stroke undergoing thrombectomy: a systematic review and meta-analysis. JAMA 2019;322:1283-93.

21 Jadhav AP, Bouslama M, Aghaebrahim A, et al. Monitored anesthesia care vs intubation for vertebrobasilar stroke endovascular therapy. JAMA Neurol 2017;74:704-9.

22 Taqi MA, Suriya SS, Sodhi A, et al. Ideal sedation for stroke thrombectomy: a prospective pilot single-center observational study. Neurosurg Focus 2019;46:E16.

23 Chan A-W, Tetzlaff JM, Altman DG, et al. SPIRIT 2013 statement: defining standard protocol items for clinical trials. Ann Intern Med 2013;158:200-7

24 Powers WJ, Rabinstein AA, Ackerson T, et al. 2018 guidelines for the early management of patients with acute ischemic stroke: a guideline for healthcare professionals from the American heart Association/ American stroke association. Stroke 2018;49:e46-110.

25 Talke PO, Sharma D, Heyer EJ, et al. Republished: Society for neuroscience in anesthesiology and critical care expert consensus statement: anesthetic management of endovascular treatment for acute ischemic stroke. Stroke 2014;45:e138-50.

26 Warner JJ, Harrington RA, Sacco RL, et al. Guidelines for the early management of patients with acute ischemic stroke: 2019 update to the 2018 guidelines for the early management of acute ischemic stroke. Stroke 2019;50:3331-2.

27 Peng Y, Li Y, Jian M, et al. Choice of anesthesia for endovascular treatment of acute ischemic stroke: protocol for a randomized controlled (canvas) trial. Int J Stroke 2017;12:991-7.

28 American Society of Anesthesiologists Task Force on S, Analgesia by $\mathrm{N}-\mathrm{A}$. Practice guidelines for sedation and analgesia by NonAnesthesiologists by the American Society of anesthesiology. Anesthesiology 2002;96:1004-17.

29 Lindsberg PJ, Mattle HP. Therapy of basilar artery occlusion: a systematic analysis comparing intra-arterial and intravenous thrombolysis. Stroke 2006;37:922-8.

30 Wang A, Stellfox M, Moy F, et al. General anesthesia during endovascular stroke therapy does not negatively impact outcome. World Neurosurg 2017;99:638-43.
31 Zhang X, Luo G, Mo D, et al. Predictors of good outcome after endovascular treatment for patients with vertebrobasilar artery occlusion due to intracranial atherosclerotic stenosis. Clin Neuroradiol 2019;29:693-700.

32 Schönenberger S, Uhlmann L, Hacke W, et al. Effect of conscious sedation vs general anesthesia on early neurological improvement among patients with ischemic stroke undergoing endovascular thrombectomy: a randomized clinical trial. JAMA 2016;316:1986-96.

33 Hulley SB CS, Browner WS, Grady D, et al. Designing clinical research : an epidemiologic approach. 75. 4th edn. Philadelphia, PA: Lippincott Williams \& Wilkins, 2013.

34 Sugg RM, Jackson AS, Holloway W, et al. Is mechanical embolectomy performed in nonanesthetized patients effective? AJNR Am J Neuroradiol 2010;31:1533-5.

35 Davis MJ, Menon BK, Baghirzada LB, et al. Anesthetic management and outcome in patients during endovascular therapy for acute stroke. Anesthesiology 2012;116:396-405.

36 Langner S, Khaw AV, Fretwurst T, et al. [Endovascular treatment of acute ischemic stroke under conscious sedation compared to general anesthesia - safety, feasibility and clinical and radiological outcome]. Rofo 2013;185:320-7.

37 Hassan AE, Akbar U, Chaudhry SA, et al. Rate and prognosis of patients under conscious sedation requiring emergent intubation during neuroendovascular procedures. AJNR Am J Neuroradiol 2013;34:1375-9.

38 Abou-Chebl A, Zaidat OO, Castonguay AC, et al. North American SOLITAIRE Stent-Retriever acute stroke Registry: choice of anesthesia and outcomes. Stroke 2014;45:1396-401.

39 Athiraman U, Sultan-Qurraie A, Nair B, et al. Endovascular treatment of acute ischemic stroke under general anesthesia: predictors of good outcome. J Neurosurg Anesthesiol 2017;30:223-30.

40 Mundiyanapurath S, Schönenberger S, Rosales ML, et al. Circulatory and respiratory parameters during acute endovascular stroke therapy in conscious sedation or general anesthesia. J Stroke Cerebrovasc Dis 2015;24:1244-9.

41 Hassan AE, Chaudhry SA, Zacharatos $\mathrm{H}$, et al. Increased rate of aspiration pneumonia and poor discharge outcome among acute ischemic stroke patients following intubation for endovascular treatment. Neurocrit Care 2012;16:246-50. 